\title{
Lagrange and the secular equation
}

\author{
Frédéric Brechenmacher
}

Published online: 21 May 2014

(c) Centro P.RI.ST.EM, Università Commerciale Luigi Bocconi 2014

\begin{abstract}
In Lagrange, the mechanical stability of the oscillations of a system of bodies was characterised by the nature of the roots of a specific algebraic equation. The latter was commonly known in the nineteenth century as the "equation to the secular inequalities in plantetary theory" because of its role in the study of the stability of the solar system. It is, in modern terms, the characteristic equation of a symmetrical linear system; its study sheds light on an important part of the history of linear algebra. In the nineteenth century, the secular equation played a pivotal role in both celestial mechanics and in geometry, in complex analysis as well as in arithmetic. This transversality shows that the work of Lagrange was fundamental for the constitution of a culture of algebra that was shared throughout Europe.
\end{abstract}

Keywords Lagrange - d'Alembert - Laplace - History of algebra $\cdot$ Linear algebra $\cdot$ Mechanics $\cdot$ Differential equations - Characteristic equations - Proper values . Matrices

\section{Introduction}

"This article analyses the works of Joseph-Louis Lagrange that played an important role in the development of matrix theory, and more generally, in linear algebra". We are tempted to introduce our article with a sentence of this sort. We could then fall back on the divisions into great mathematical theories or disciplines in order to delimit the terrain of historical research. However, whether we are concerned with analysing the works of Lagrange in

F. Brechenmacher $(\square)$

Département humanités et sciences sociales, École

Polytechnique, 91128 Palaiseau Cedex, France

e-mail: frederic.brechenmacher@polytechnique.edu "probability theory" or "number theory" or "fluid mechanics", in "algebra", etc., these categories pose problems, since the significations they have embraced have changed over time. In Lagrange's day, these categories all fell into the field of "mathematical sciences", while today they appertain to different disciplines. As for linear algebra and matrix theory, these simply did not exist.

Instead of concerning ourselves with the great theories, we propose here to delimit our area of investigation to the most minute level on the scale, the problem of "small oscillations". Furthermore, the problem itself will be examine in the technical details, since it is at this microhistorical level that we are best able to grasp Lagrange's originality and creativity.

In the simplest version of the oscillations of a pendulum, we are dealing with a problem that we might label as relevant to "dynamics", but in the first memoir that Lagrange devoted to this topic in 1766 , the problem is treated in the more general framework of the oscillation of a system of $n$ bodies and categorised as relating to "integral calculus". The oscillations are effectively mathematised by a system of linear differential equations of constant coefficients that are to be integrated. Lagrange is the first to give the general solution of such a system, showing that it is deduced directly from the factorisation of a particular kind of algebraic equation. A few years later, Lagrange transfers this same mathematisation to the study of the small oscillations of planets, called "secular inequalities". The problem thus passes to "celestial mechanics" and the algebraic equation that gives the solution is by then designated by the name of "secular equation". The stability

\footnotetext{
${ }^{1}$ In today's terminology, the secular equation corresponds to the "characteristic equation" of a symmetrical matrix with real coefficients.
} 
of the solar system depends on the nature of the roots of the equation. In 1788, the problem of small oscillations occupied a place of privilege in the Mécanique analitique [19], where it is the first example of an application. It would be a source of inspiration for the entire course of the nineteenth century for works in astronomy, geometry, arithmetic, algebra, analysis, fluid mechanics, elasticity, the theory of light, and more.

The problem of "small oscillations" plays such a large part in the divisions into great theories that it gives us the opportunity to take an original look at the relationships between the different branches of the mathematical sciences. In particular, it allows us to shed new light on the "algebraic" dimension of Lagrange's work.

Historiography has insisted at length on "Lagrange's algebraic style" [6, 7], described as a dual reduction of mechanics to analysis, and analysis to algebra [23-25]. But of all of the categories that can be used to divide the mathematical sciences, the category of "algebra" is not the least problematic.

With regard to the time of Lagrange, it is particularly necessary to distinguish between "algebra" and "algebraic". Algebra, on one hand, was generally considered as the science of solving equations. In the works that Lagrange devoted to it, he distinguished three principal types of problems: the solvability by roots of a general equation of given degree; the study of particular equations, and finally the approximate solution of numerical equations. The works on the general problem of solvability are famous for having inspired the research of Évariste Galois. The particular equations often pass by unnoticed in historiography, yet the new approaches that Lagrange developed in order to study the "secular equations" form the basis of linear algebra, a mathematical discipline that would be constituted in the 1930s and that since then has played a structural role in the organisation of scientific knowledge.

But let's return to our distinction between "algebra" and "algebraic". Historiography often associates the "algebraic" dimension of Lagrange's work to the "formal" nature of an approach detached from geometric intuition and from the physical observation to the advantage of symbolic writing:

The implementation of the calculus of variations necessitates a new form of intuition, in the sense that it is completely detached from the consideration of the properties of geometric figures, because it operates exclusively within a framework of algebraic formulas. ...The introduction of mathematical formalism into dynamics signifies the imposition of an aesthetic algebraic form on the study of the science of motion, which means the abandonment of all means other than that of algebraic calculation ([1], p. 134 (our trans.)).

We will have to qualify this classic description of Lagrange's style of mathematisation In effect we will see in this present article that the mathematisation of the problem of small oscillations is never reduced to a formalism and that the algebraic practices are inseparable from mechanical interpretations. These mixed interpretations play an essential role in Lagrange's creativity, but they also limited his impact and lie at the basis of an error that was propagated for almost a century thanks to the great posterity of Lagrange's works on the secular equation.

\section{From the small oscillations of a string to the secular inequalities of the planets}

Lagrange began his work on small oscillations towards the end of his period in Turin. In 1766, the year of his departure for Berlin, he published his "Solutions de différents problèmes de calcul intégral" [15], the last of a series of his contributions to the Miscellanea Taurinensia, the journal of the Turin Academy of Sciences, which he had helped found in 1758.

"Solutions" deals with mathematical questions tied to issues of mechanics. Lagrange drew on the work of several scientists with whom he corresponded, such as Leonhard Euler and Daniel Bernoulli, but the author who most influenced his approach is undoubtedly Jean le Rond d'Alembert. The two scientists had commenced their friendship during Lagrange's first visit to Paris in 1763. At the time, their epistolary correspondence was largely devoted to the problem of vibrating strings. The problem that we will deal with in this present article is a particular case: it involves describing, as a function of time $t$, the small oscillations $\xi(t)$ of a string fixed at one of its ends, and weighted with a certain number of bodies.

In order to analyse this problem by the same route that d'Alembert and Lagrange followed, we will go forward by successive generalisations, from the study of a simple pendulum to that of the oscillations of two bodies and then any number $n$ of bodies.

\subsection{The case of the pendulum}

The problem of the pendulum was already a classic by Lagrange's day. Its movement is mathematised by the following equation, which includes a constant parameter $\alpha$ :

$\frac{d^{2} \xi}{d t}=\alpha \xi$

This type of equation is called a linear second-order differential equation with constant coefficients. The 
solution to it was given, among others, by Euler in 1743 , and is based on an analogy between the second-order differential equation and the following algebraic seconddegree equation,

$x^{2}=\alpha$,

in which the two roots, $\sqrt{\alpha}$ and $-\sqrt{\alpha}$ give the parameters of the family of solutions sought:

$\xi(t)=A e^{\sqrt{\alpha} t}+B e^{-\sqrt{\alpha} t}$

(with constants $A$ and $B$ depending on the initial conditions of the problem).

We observe that the "algebraic nature" of the roots of the Eq. (*) is intrinsically related to the "mechanical nature" of the oscillations of the pendulum:

1. if the roots $\sqrt{\alpha}$ and $-\sqrt{\alpha}$ are real and distinct ( $\alpha$ is strictly positive), then the oscillations $\xi(t)$ grow exponentially in function of time $t$ : they are thus instable;

2. in the case where the roots are complex and distinct $(\alpha$ is strictly negative), the exponential parts compensate for each other and the solutions can be expressed in the following trigonometric form:

$\xi(t)=A \cos (\sqrt{-\alpha} t)+B \sin (\sqrt{-\alpha} t)$.

The oscillations are then bounded; they are the result of two periodic oscillations of which the period depends on $\sqrt{-\alpha}$;

3. finally, the case where the roots are equal poses particular problems to which we will return later.

While the third case may appear insignificant, in reality it plays a crucial role and caused problems for mathematicians for almost a century. This situation will lead us to discuss the tension between generalities and particularities in the dynamics of mathematical knowledge.

\subsection{D'Alembert: the two-body system}

In the eighteenth century, the pendulum problem served as a model for the study of various mechanical systems. D'Alembert in particular generalised the description of small oscillations of a string "loaded with two weights and at an infinitely small distance from the vertical", , a problem that Daniel Bernoulli believed to be too complex for the methods of analysis.

The success of this generalisation led d'Alembert to affirm the general importance of the principle of virtual velocities, which was the central principle for his unification of solid-body mechanics in the Traité de dynamique [8] of $1743^{3}$. This principle makes it possible to obtain the

\footnotetext{
${ }^{2}$ For more on this, see $[10,11]$.
}

differential equations that mathematise the oscillations $\xi$. In order to simplify these equations, d'Alembert suppresses all the non-linear terms, of the type $\xi^{2}, \xi^{3}, \xi^{4}$, for the reason that these are negligible since the oscillations $\xi$ themselves are already assumed to be very small. The determination of the oscillations $\xi_{1}(t)$ and $\xi_{2}(t)$ of two weights is thus reduced to the study of a system of two second-order differential equations with constant coefficients:

$\left\{\begin{array}{l}\frac{d^{2} \xi_{1}}{d t}=A_{11} \xi_{1}+A_{12} \xi_{2} \\ \frac{d^{2} \xi_{2}}{d t}=A_{21} \xi_{1}+A_{22} \xi_{2}\end{array}\right.$.

D'Alembert sought to express this system under the form of two independent equations of the type (1), each of which would be integrable separately (see Sect. 1.1). We are thus dealing with the search for the particular solutions, $\xi_{1}^{\prime}$ and $\xi_{2}^{\prime}$, obtained by a modification of the initial variables $\xi_{1}$ and $\xi_{2}$, and permitting the reduction of the system (2) to a simple situation of proportionality:

$\left\{\begin{array}{l}\frac{d^{2} \xi_{1}^{\prime}}{d t}=S \xi_{1}^{\prime} \\ \frac{d^{2} \xi_{2}^{\prime}}{d t}=S \xi_{2}^{\prime}\end{array}\right.$

What are the possible values for the coefficient of proportionality $S$ ? It must be verified that:

$\left\{\begin{array}{l}A_{11} \xi_{1}^{\prime}+A_{12} \xi_{2}^{\prime}=S \xi_{1}^{\prime} \\ A_{21} \xi_{1}^{\prime}+A_{22} \xi_{2}^{\prime}=S \xi_{2}^{\prime}\end{array}\right.$

or again:

$\left\{\begin{array}{l}\left(A_{11}-S\right) \xi_{1}^{\prime}+A_{12} \xi_{2}^{\prime}=0 \\ A_{21} \xi_{1}^{\prime}+\left(A_{22}-S\right) \xi_{2}^{\prime}=0\end{array}\right.$

For each value of $S$, one thus obtains a linear system of two equations from which one can deduce the values of $\xi_{1}^{\prime}$ and $\xi_{2}^{\prime}$. Now, since the system $\left(2^{\prime}\right)$ is linear, we can note that if $\left(\xi_{1}^{\prime}, \xi_{2}^{\prime}\right)$ is a pair of solutions, then so is $\left(2 \xi_{1}^{\prime}, 3 \xi_{2}^{\prime}\right)$, and more generally, so are all pairs $\left(a \xi_{1}^{\prime}, b \xi_{2}^{\prime}\right)$, with $a$ and $b$ any real numbers. Today we would say that the solutions of a linear system form a vector space of dimension 2. That notion did not exist at the time of d'Alembert and Lagrange; their work played a strong role in its development.

It is thus necessary to seek the values of $S$ for which system $\left(2^{\prime \prime}\right)$ has an infinite number of solutions. Now, it is for this necessity that the two equations that make up the system are to be proportional, that is, that their cross product—also called the determinant—is null:

\footnotetext{
3 In today's terminology, this is about calculating the Lagrangian $L=T-V$ where $T$ is the kinetic energy and $V$ the potential energy.
} 
$\left|\begin{array}{cc}A_{11}-S & A_{12} \\ A_{21} & A_{22}-S\end{array}\right|=0$

This condition gives a second-degree equation in $S$ :

$\left(A_{11}-S\right)\left(A_{22}-S\right)-A_{21} A_{12}=0$

or again:

$S^{2}\left(A_{11}+A_{22}\right) S+A_{11} A_{22}-A_{21} A_{12}=0$.

We will call the roots of this equation $\alpha_{1}$ and $\alpha_{2}$. The solutions to the problem are thus given by the linear combination of the solutions of the two independent equations as follows:

$\left\{\begin{array}{l}\frac{d^{2} \xi}{d t}=\alpha_{1} \xi \\ \frac{d^{2} \xi}{d t}=\alpha_{2} \xi\end{array}\right.$,

which can be integrated by the method of Sect. 1.1:

$\xi_{1}^{\prime}(t)=A_{1} e^{\sqrt{\alpha_{1}} t}+B_{1} e^{-\sqrt{\alpha_{1}} t}$.

$\xi_{2}^{\prime}(t)=A_{2} e^{\sqrt{\alpha_{2}} t}+B_{2} e^{-\sqrt{\alpha_{2}} t}$

Finally, the set of all solutions of the problem is obtained by the combination of the two particular solutions above: the problem is solved.

We see that this solution is reduced, as in the case of the simple pendulum, to the consideration of an algebraic equation. Furthermore, the mechanical nature of the oscillations depends here again on the algebraic nature of the roots of this equation. But the Eq. (*) of the simple pendulum does not play the same role as the Eq. (**). This "equation in $S$ " has in effect the function of separating the linear system (2) in order to reduce it to two independent equations $\left(2^{\prime \prime \prime}\right)$, that is, a simple situation of proportionality. Now, such a reduction appears to necessitate that the two roots of the equation in $\mathrm{S}$ are distinct. The occurrence of a double root thus appears once again as a particular case: in the case where $\alpha_{1}=\alpha_{2}$, d'Alembert's method seems to fail to decompose the system into two independent equations. We will come back to this later.

\subsection{Lagrange: the general case}

In spite of the assertions of generality, d'Alembert did not arrive at treatment of the case of a string loaded with any two masses, or with three equal masses. In his last work of the Turinese period, Lagrange intended to give a

méthode générale pour déterminer le mouvement d'un système quelconque de corps qui agissent les uns sure les autres, en supposant que ces corps ne fassent que des oscillations infiniment petites autour de leurs points d'équilibre ([15], p. 519).

(general method for determining the movement of any system of bodies that act on each other, presuming that those bodies make oscillations that are infinitely small about their equilibrium point).

20 years later, Lagrange would assign a central place to this problem in the architecture of his Mécanique analitique. The problem of small oscillations opens the part of applications of the "general principles" on which Lagrange based his Mécanique. The "simplicity" and the "generality" of its solution contrasted with the long-time difficulties and confusion raised by the study of vibrating strings. The problem of small oscillations thus comes to support the role that Lagrange intended to play in the history of mechanics:

La dynamique est la science des forces accélératrices ou retardatrices, \& des mouvements variés qu'esses peuvent produire. Cette science est due entièrement aux Modernes, \& Galilée est celui qui en a jeté les premiers fondements. ... La Mécanique devint une science nouvelle entre les mains de Newton, \& ses Principes Mathématiques qui parurent pour la première fois en 1687, furent l'époque de cette révolution .... Enfin l'invention du calcul infinitésimal mit les Géomètres en état de réduire à des équations analytiques les lois du mouvement des corps; \& la recherche des forces \& des mouvements qui en résultent est devenue depuis le principal objet de leurs travaux. Je me suis proposé ici de leur offrir un nouveau moyen de faciliter cette recherche ... ([19], pp. 158-159).

(Dynamics is the science of accelerating or retarding forces, and of the various movements that these can produce. This science is due entirely to the Moderns, and Galileo is the one who laid the first foundations. ... Mechanics became a new science in the hands of Newton and his Principia Mathematica, which appeared for the first time in 1687, the time of that revolution. ... Finally the invention of integral calculus put Geometry in a condition to reduce to those analytical equations the laws of the movement of bodies; and the research in the forces and the movements that resulted has been ever since the principal object of their works. I have proposed myself here to offer them a new way of facilitating that research).

This dual ideal of generality and simplicity would strongly mark the treatment of the problem of small oscillations for more than a century. It implies the 
consideration of a linear system constituted of any number $n$ of linear differential equations with constant coefficients $A_{i, j}$ :

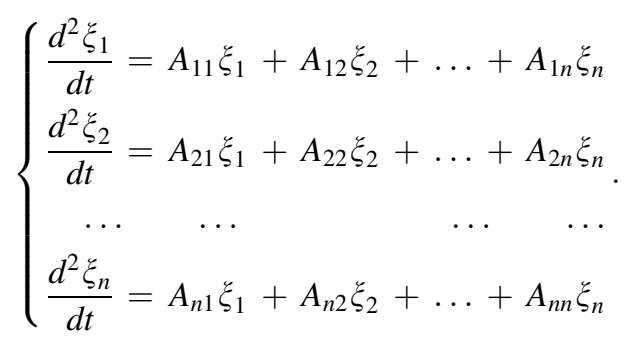

Lagrange's approach directly generalises that of d'Alembert: the integration of the system is effectively obtained by its decomposition into $n$ independent equations, in which the parameters are the roots of an algebraic equation of degree $n$, the "equation in $S$ ". To each of its roots $\alpha_{1}$ is associated the solution

$\xi^{\prime}(t)=A e^{\sqrt{\alpha} t}+B e^{-\sqrt{\alpha} t}$

(with $A$ and $B$ both constant). In the case where the $n$ roots are distinct, we obtain $n$ independent solutions. Beginning with the linear combinations of which we can express all the $n$ solutions of system (3) by explicit analytical expressions.

Lagrange also discovered how to express the solutions directly by means of simple factorisations of the "equation in $S^{\prime \prime}$. Thus he did not limit himself to a generalisation of d'Alembert's research. Instead, he gave a completely new solution to the mechanical problem of small oscillations that he reduced to a simple "analytical expression", that is, to a formula. We note that Lagrange preferred the term "analytical expression" to that of "algebraic formula". The distinction is important, because the recourse to polynomial expressions is not associated to algebra but rather to analysis, envisioned as a general method of solving mechanical problems.

\section{Algebra and mechanics: Lagrange's principle of stability}

The approach taken by d'Alembert and Lagrange to the study the problems of small oscillations is based on the close relationship between the algebraic and mechanical interpretations. In fact, the situation presented here is far from limited to the formalisation of a concrete problem. To

\footnotetext{
${ }^{4}$ In today's language, the method consists in giving a general polynomial expression of the eigenvectors of a symmetrical matrix A as quotients of the minors extracted from the characteristic determinant $|A-S I|$ and by a factorisation of this by means of a linear term: $|A-S I| /\left(S-\alpha_{i}\right)$ (where $\alpha_{i}$ is a root of the characteristic equation $|A-S I=0\rangle$. Such an expression is identified with a factor close to the non-null columns of the matrix of $|A-S I|$. See [2].
}

the contrary, the mechanical interpretations are inseparable from the creativity involved in solving the problem of small oscillations, and in particular from the algebraic procedure that played a key role: the decomposition of a linear system by means of the factorisation of the equation in $S$.

This algebraic practice itself came from the mathematisation of a mechanical observation according to which the oscillations of a string weighted with $n$ masses can be decomposed into independent oscillations of $n$ strings weighted by a single mass. D'Alember remarked:

Je me contenterai de dire que l'one remarque aisément ... la double oscillation que M. Bernoulli a observée dans le mouvement du pendule dont il s'agit; ... d'où il est facile de voir que les mouvements des corps M,m sont composées de deux mouvements, synchrones chacun à celui d'un pendule simple ([9], p. 182).

(I will content myself with saying that one easily notices ... the double oscillation that $\mathrm{Mr}$ Bernoulli has observed in the movement of the pendulum we are dealing with ... from which it is easy to see that the movements of the bodies $\mathrm{M}, \mathrm{m}$ are composed of two movements, each synchronised with that of a simple pendulum).

We thus need to seek $n$ "proper oscillations" $\alpha_{i}$ each corresponding to the period of a simple pendulum, and in which the linear combinations give the set of solutions of the system of $n$ linear differential equations. In today's terminology, this procedure consists in the determination of the base of a vector space of dimension $n$ given by the eigenvectors $\xi_{i}$ associated with the eigenvalues $\alpha_{i}$ of the differential system. But in the work of Lagrange the algebraic and mechanical interpretations are never separated from each other: the $\alpha_{i}$ are simultaneously the roots of an algebraic equation of degree $n$ and the beats of the oscillations of the simple pendulum. Lagrange wrote:

Ainsi le mouvement des corps sera le même, dans ce cas que s'ils étaient pesants et qu'ils fussent suspendus chacun à un fil ..., d'où l'on voit que le système est susceptible d'autant de différents mouvements isochrones que l'équation $\mathrm{P}=0$ a de racines réelles négatives et inégales ([15], p. 534).

(Thus the movement of the bodies will be the same, in the case that they are heavy as when they are suspended by a string ... from which one sees that the system is capable of so many different isochronous movements that the equation $P=0$ has real negative and distinct roots).

The mechanical stability of the oscillations is thus characterised by Lagrange by the algebraic nature of the 
root of the equation in $\mathrm{S}$, according to whether they are all distinct and real (case 1), all distinct and imaginary (case 2) or finally that multiple roots intervene (case 3):

De là on tire une méthode générale pour voir si l'état d'équilibre d'un système donné de corps est stable, c'est-à-dire si, les corps étant infiniment peu dérangés de cet état, ils y reviendront d'eux-mêmes, ou au moins tendront à y revenir...

$1^{\circ} \mathrm{Si}$ toutes le racines de cette équation son réelles négatives et inégales, l'état d'équilibre sera stable en général, quel que soit le dérangement initial du système;

$2^{\circ} \mathrm{Se}$ ces racines sont toutes réelles, positives ou toute imaginaires ou en partie positives, et en partie imaginaires, l'état d'équilibre n'aura aucune stabilité, et le système une fois dérangé de cet état ne pourra le reprendre;

$3^{\circ}$ Enfin, si les racines sont en partie réelles, négatives et inégales, et en partie réelles négatives et égales ou réelles et positives, ou imaginaires, l'état d'équilibre aura seulement une stabilité restreinte et conditionnelle ([15], p. 352).

(From this is deduced a general method for seeing if the state of equilibrium of any system of bodies is stable, that is, if, once that the bodies are disturbed infinitely little from this state, they will return to it, or at least tend to return to it.

1. If all the roots of this equation are real negative and distinct, the equilibrium state will be generally stable, whatever the initial disturbance of the system;

2. If these roots are all real positives, or all imaginary, or in part positive and in part imaginary, the equilibrium state will have no stability, and the system, once disturbed from this state, will not be able to return to it;

3. Finally, if the roots are in part real negative and distinct, and in part real negative and equal, or real and positive, or imaginary, the equilibrium state will only be a restricted or conditional stability).

\section{The secular equation}

In the 1770s, Lagrange transferred his mathematisation of small oscillations of a string to the study of the small oscillations of the planets. Although very slight, the oscillations called "secular" not only hinder the calculation of the ephemerides in the long term, but over long periods can also produce significant modifications in the orbits and thus threaten the stability of the solar system. In the words of Lagrange:
Si les Planètes étaient simplement attirées par le Soleil, et n'agissaient point les unes sur les autres, elles décriraient autour de cet astre, des ellipses variables suivant les lois de Kepler, comme Newton l'a démontré le premier, et une foule d'Auteurs après lui. Mais les observations ont prouvé que le mouvement elliptique des Planètes est sujet à des petites oscillations, et le calcul a démontré que leur attraction mutuelle peut en être la cause. Ces variations sont de deux espèces: les unes périodiques et qui ne dépendent que de la configuration des Planètes entre elles; celles-ci sont le plus sensibles, et le calcul en a déjà été donné par différents Auteurs; les autres séculaires et qui paraissent aller toujours en augmentant, ce sont le plus difficiles à déterminer tant par les observations que par la Théorie. Les premières ne dérangent point l'orbite primitive de la Planète; ce ne sont, pour ainsi dire, que des écarts passagers qu'elle fait dans sa course régulière, et il suffit d'appliquer ces variations au lieu de la Planète calculé par les Tables ordinaires du mouvement elliptique. Il n'en est pas de même des variations séculaires. Ces dernières altèrent le éléments mêmes de l'orbite, c'est-à-dire la position et la dimension de l'ellipse décrite par la planète; et quoique leur effet soit insensible dans un court espace de temps, il peut néanmoins devenir à la longue très considérable ([17], p. 125).

(If the planets are simply attracted by the sun, and they do not act at all upon each other, they describe about that star variable ellipses according to Kepler's laws, as Newton had proved for first, and a crowd of authors after him. But the observations have proven that the elliptical movement of the planets is subject to small oscillations, and calculations have shown that their mutual attraction might be the cause. These variations of are two kinds: one is periodic and depends on the configuration of the planets among themselves; these are the most sensitive, and the calculations already been done by different authors; the others are secular and a appear to be constantly increasing, they are the most difficult to determine with regard to both observation and theory. The first of these do not at all disturb the primitive orbit of the planet; they are only, we might say, temporary deviations that it makes in its regular course, and it is sufficient to apply those variations in place of the planet calculated by the ordinary tables of elliptical movement. It is not at all the same with the secular variations. These alter the elements themselves of the orbit, that is, the position and the dimension of the ellipse described by the planet; and even though their effect is indiscernible in a short space of time, it can 
nevertheless become very considerable over the long term).

From 1774 Lagrange transferred to secular inequalities the approach that he had elaborated in 1766 for the case of vibrating strings. This méthode fort ingénieuse (very ingenious method) strongly impressed Pierre Simon de Laplace, who immediately adopted it:

J'ai donné dans un autre Mémoire ... les expressions des inégalités séculaires des planètes ... mais le peu d'utilité de ce calcul pour les besoins de l'Astronomie, joint aux difficultés qu'il présentait, m'avait fait abandonner cette idée, et j'avoue que je ne l'aurais pas reprise, sans la lecture d'un excellent Mémoire Sur les inégalités séculaires du mouvement des nœuds et de l'inclinaison des orbites des planètes que M. de Lagrange vient d'envoyer à l'Académie, et qui paraîtra dans un des Volumes suivants. Cet illustre géomètre, au moyen d'une transformation heureuse, réduit le problème à l'intégration d'autant d'équations linéaires du premier ordre qu'il y a d'inconnues; il donne ensuite une méthode fort ingénieuse pour les intégrer, et pour déterminer les constantes que renferme l'intégrale, quelque soit le nombre de planètes ([20], p. 354).

(I have given in another memoir ... the expressions of the secular inequalities of the planets ... but the scant utility of the calculation for the requirements of astronomy, joined to the difficulties that it presents, made me abandon these ideas, and I would never have taken them up again, without the reading of an excellent memoir "On the secular inequalities of the movement of the nodes and the inclination of the orbits of the planets" that Mr Lagrange has sent to the Academy, and which will appear in one of the coming volumes. This illustrious geometer, by means of a fortunate transformation, reduced the problem to the integration as many first-order linear equations as there are unknowns; he then gives a very ingenious method for integrating and determining the constants contained in the integral, whatever the number of planets).

In the years from 1770 to 1780, Lagrange and Laplace devoted numerous memoirs to the study of the secular inequalities. These related works nourished the two great treatises of synthesis: Lagrange's Mécanique analytique and Laplace's Traité de mécanique céleste [21].

The implications of these works regarding the long-term behaviour of the solar system had a considerable impact on the practice elaborated by Lagrange for decomposing linear systems by factorisation of the equation in $S$. Thus in the nineteenth century this equation was often referred to as the one "à l'aide de laquelle on détermine les inégalités séculaires des mouvements des planètes" (the equation to the secular inequalities in planetary theory" [5]), which we will abbreviate here as the "secular equation".

\subsection{The renewal of the problem of stability}

We have seen that the practice of the decomposition of linear systems intrinsically interweaves algebraic and mechanical significations. Now, on the level of mechanics, the situation of the secular inequalities is never the same as that of the oscillations of a string. In the second case, the oscillations are presumed "always extremely small, by nature of the problem" [9, p. 167 (our trans.)], and the roots of the equation in $S$ must therefore be real, negative and distinct. However, in the case of the planets, the stability of the solar system is never assured. To the contrary, the question involved here is crucial. Now, according to the criteria established by Lagrange, the occurrence of imaginary or multiple roots implies the presence of the "secular terms", that is, unbounded oscillations, liable to destroy the stability of the solar system in the long term. Lagrange wrote:

Avant de terminer cet Article, nous devons encore remarque que, quoique nous ayons supposé que les racines de l'équation [en $\mathrm{S}$ ] soient réelles et inégales, il peut néanmoins arriver qu'il y en ait d'égales ou d'imaginaires; ... dans l'un et l'autre case, les quantités dont il s'agit croîtront à mesure que t croît; ... mais heureusement ces cas ne paraissent pas avoir lieu dans le Système du monde ([16], p. 665).

(Before concluding this article, we must still remark that, although we suppose that the roots of the equation [in $S$ ] are real and distinct, it can nevertheless occur that there are [roots that are] equals or imaginaries. ... In either case, the quantity concerned would grow in the measure that $t$ grows; ... but fortunately this case appears never to take place in the system of the world).

The transfer to the planets of the mathematisation of vibrating strings thus led to a new challenge: to prove that the roots of the secular equation are always real and distinct. But this objective collided with the great generality at which Lagrange aimed. For any given number of bodies, the secular equation is of $n$ degrees, and cannot thus be solved by radicals if $n \geq 5$. Further, one cannot even write this equation in its explicit polynomial form. Still less can one express the roots using the analytical expressions that Lagrange favoured.

Lagrange initially settled on an approximate solution to the problem. In 1781 he also dealt with the question of the 
stability of the solar system and treated the orbits of Saturn and Jupiter separately from those of Mars, Earth, Venus and Mercury. The problem is then reduced to the effective calculation of the roots of two equations of degrees 2 and 4. From a theoretical point of view, Lagrange analysed these algebraic equations based on his method of reduction of degree by substitutions, famous for having inspired the work of Galois ([17], p. 311). But Lagrange also proposes a numerical application: with the aid of tables of astronomical observations, he shows that the approximate values of the six roots are indeed real and distinct.

\subsection{A particular expedient}

For Lagrange, however, an approximate solution did not remove the "doubts" regarding the stability of the solar system, because the numerical calculation of the values of the roots is dependent on the values attributed to the masses of the planets and the astronomical tables. In order to eliminate doubts a genuine mathematical proof was needed. But the generality of the problem forced the abandonment of hope of obtaining an analytical expression of the roots of the secular equation in order to orient itself instead towards, in Lagrange's words, an "artifice particulier" (particular expedient):

Mais, comme les racines que nous venons de trouver dépendent des valeurs supposées aux masses des Planètes, on pourrait douter si, en changeant ces valeurs, on ne tomberait peut être pas dans les racines égales ou imaginaires. Pour lever tout à fait ce doute, il faudrait pouvoir démontrer, en général, que, quelles que soient les valeurs des masses, pourvu seulement qu'elles soient positives, le racines de l'équation dont il s'agit sont toujours réelles et inégales. Cela est facile lorsqu'on ne considère à la fois que l'action mutuelle de deux Planètes ...; mais cette équation se complique et s'élève à mesure que le nombre des Planètes augmente; c'est pourquoi il devient de plus en plus difficile da juger à priori de la qualité des racines. Cependant il ne parait pas impossible de parvenir, par quelque artifice particulier, à décider cette question d'une manière générale; et comme c'est un objet également intéressant pour l'Analyse et pour l'Astronomie physique, je me propose de m'en occuper. En attendant, je me contenterai de remarque que, dans le cas présent, les racines trouvées son trop différents entre elles pour qu'un petit changement dans les masses adoptées puisse les rendre égales, et encore moins imaginaires ([18], p. 316).

(But, as the roots that we have just found depend on the presumed values of the masses of the planets, one might harbour a doubt whether, in changing these values, one might not fall into roots that are equal or imaginary. To remove this doubt completely, it would be necessary to prove, in general, that whatever the values of the masses, the roots of the equation concerned are always real and distinct. This is easy when one considers only the mutual action of two planets ...; but this equation becomes complicated and raised in degree as the number of planets increases; this is why it become more and more difficult to judge a priori the quality of the roots. Nevertheless it does not seem impossible to arrive, by means of a particular expedient, to decide this question in a general manner; and as this is an object of interest for both analysis and physical astronomy alike, I intend to address it. In the meantime, I will content myself with remarking that, in the present case, the roots found are too different from each other for a small change in the masses adopted to render then equal, much less imaginary).

Laplace never stopped searching for the expedient which would make it possible to prove the stability of the system of the world and, through it, that Lagrange's method permitted prediction of the positions of the heavenly bodies over an "extremely long" interval":

Ces valeurs ne peuvent servir que pour un temps limité, après lequel, les excentricités devenant fort grandes, la supposition qu'elles sont peu considérables et d'après laquelle elles ont été trouvées cesse d'être exacte; on ne peut donc étendre à un temps quelconque les résultats obtenus dans cette supposition, qu'autant que l'on est assuré que les racines de l'équation [séculaire] sont toutes réelles et inégales; mais il serait très difficile d'y parvenir par la considération directe de cette équation ([19], p. 464). (These values can only serve for a limited time, after which, the eccentricities having become very large, the supposition that they are of little account and by means of which they have been found, ceases to be exact; one cannot thus extend to any [length of] time the results obtained from this supposition; except to the extent that one can be assured that the roots of the [secular] equation are real and distinct, but that would be very difficult to arrive to by the direct consideration of this equation).

\footnotetext{
${ }^{5}$ Let us recall that Laplace and Lagrange disregarded the terms that depend on the square of the masses of the planets. This linear approximation limits the duration of the validity of their predictions, as was shown in particular by Le Verrier in 1856. The question of whether the calculation of the secular variations could be extended to an indeterminate period was hotly debated in the nineteenth century, before finally being answered in the negative by Poincaré. For more on this subject, see [22, pp. 184-187].
} 
The expedient so ardently sought was finally discovered by Laplace in 1789 . It derives from a property uncovered in Lagrange's works of 1774: the linear systems (3) involved in the mechanical problems of small oscillations are far from being arbitrary; their coefficients are mirrored on each side of the diagonal: $A_{12}=A_{21}, A_{13}=A_{31}$, etc. ${ }^{6}$ Today we would say that such systems are symmetrical.

By linking this property to mechanical interpretations of the constancy of angular momentum, Laplace proved that the roots of the secular equation are always real. This led him victoriously to conclude the stability of the system of the world.

\section{The culture of algebra created by the secular equation}

Laplace's proof might appear to conclude the discussion carried out by d'Alembert and Lagrange about the nature of the roots of the secular equation. Nothing of the sort. To the contrary, we find, over the entire course of the nineteenth century, a great number of references to the secular equation. This references are rarely concerned with celestial mechanics. Thus there is a memoir by Augustin Louis Cauchy of 1829, entitled "Sur l'équation à l'aide de laquelle on détermine les inégalités séculaires des planètes" [5], which is devoted to the analytic geometry of conics and quadratics. James Joseph Sylvester chose, in 1852, [27] to publish in French his pioneering works on matrix theory under the title "Sur une propriété nouvelle de l'équation qui sert à déterminer les inégalités séculaires des planètes". We can also cite the algebraic formulation used by Charles Hermite in 1857 [13] in a memoir devoted to the representation of an integer as the sum of four squares.

In fact, beginning with Siméon Denis Poisson's work of 1808 , the question of the stability of the solar system was most frequently treated through a consideration of the nonlinear terms in the equations of dynamics. The linear approximation at the heart of the approaches of Lagrange and Laplace were thus quickly rendered obsolete, until Poincaré reclaimed them at the end of the nineteenth century [4]. The legacy of Lagrange's works on small oscillations was thus not limited to the reception of a method in a given theoretical framework. This situation is not surprising. Like all great treatises, the Mécanique analytique was the object of multiple and fragmentary readings in different times and cultural environments. In consequence, we must change the scale of analysis and turn our attention to the collective dimensions of the readings of Lagrange's works in the long term.

\footnotetext{
6 That symmetry derives from the fact that the kinetic energy and the potential energy are given by quadratic forms.
}

5.1 A practice that transverses various theoretical frameworks

As a result of Lagrange's works, the practice of decomposing linear systems through the factorisation of the secular equation was used to study other problems of mechanics in addition to those of vibrating strings and celestial mechanics: the rotation of a solid body; the study of ellipsoid deformation in the theory of elasticity; Fresnel's theory of light; Fourier's works on heat; fluid mechanics, and more. Further, beginning in the $1820 \mathrm{~s}$, the number of mathematical formulations related to the secular equation multiplied: Sturm's theorem on the number of real roots of an algebraic equation; the principal axes of conics and quadratics in analytic geometry; the theory of remainders in complex analysis; the algebraic theory of quadratic forms; matrix theory; group theory, and others.

Even though they appertain to quite different theoretical frameworks, these works are not at all independent from one another. To the contrary, they often refer to each other and thus form a coherent network of texts: In what follows we will give a brief overview of this network. All of the texts are related to Lagrange's Mechanique analytique, which shows itself to be the founding text of the questions tied to the "secular equation".

\subsection{A common culture of algebra based on the works of Lagrange}

By all evidence, the signification of the term "secular equation" was no longer the same at the beginning of the nineteenth century as it had been in the mid-eighteenth century. In fact, this term was quickly used to make reference to the specific algebraic practice developed by Lagrange, and was no longer tied to the secular inequalities of celestial mechanics. The expression "secular equation" thus came to be progressive employed to identify an algebraic culture shared on a European level and based on Lagrange's Mechanique analytique [2]. We will thus indicate below its principal characteristics: procedures, ideals, organisation of knowledge and specific values.

We first find at the heart of this common culture specific algebraic procedures, those formulated by Lagrange to decompose linear systems. But the knowledge brought to algebra by the secular equation was not limited to technical procedures. Those procedures were in effect inseparable from an ideal of generality that consisted in treating situations of $n$ variables. This generality itself is part of the particular nature of the secular equation: the equation being of degree $n$, it cannot be solved by radicals but the real nature of its roots can nevertheless be deduced by a "particular expedient", the symmetry of linear systems that engender it. 
For the entire course of the nineteenth century, this particular equation would lead to analogies and permit transfers between different branches of the mathematical sciences. The culture of the secular equation is thus accompanied by a specific organisation of mathematical knowledge. We have already mentioned the mechanical representation underlying Lagrange's technique of decomposition. Later, in 1829, Cauchy relied on analogies between the secular equation and the characteristic equations of the conics to interpret these techniques in terms of changes in reference points in analytic geometry. Later still, these same procedures served as models for the small oscillations of the theory of light, they were included in the calculation of remainders in complex analysis, in Sturm sequences of an algebraic equation, in equivalence classes of pairs of quadratic forms, and more.

The culture of the secular equation also led to specific values. A great deal of importance was attributed to the problems posed by multiple roots. In fact, the multiplicity of algebraic roots gave rise to problems as delicate as that of the occurrence of imaginary roots. These two problems are thus related, and both play a part in the development of the notion of complex numbers. It is in effect to prove the real nature of the roots of the secular equation independent of their multiplicity that Cauchy devised an instrument that was key for the emergence of complex analysis: the calculation of remainders.

\subsection{The problem of multiple roots}

We have seen in the first part of this article how a certain model for the interface between physics and mathematics was transferred by analogy to the oscillations of a pendulum, vibrating strings, and then planets. While this analogy played an essential role in the Lagrange's creation of his technique of decomposing linear systems, it also limited its impact by creating an error that was perpetuated for more than a century in the bosom of the culture of the secular equation.

To understand this, we must first go back to the situation of a simple pendulum. We have seen that the parameters of the solutions depends on the roots of a second-degree algebraic equation, Eq. (*) given earlier. Now, the generalisation of the problem of the oscillations of two bodies also requires the consideration of a second-degree equation: the secular equation. Today, these equations are both referred to as "characteristic equations". They never, however, play the same role: the first is associated with a differential equation of order 2 , the other with a system of two equations. But the role as model played by the situation of the pendulum has caused the two equations to be confused. When he states that "bodies displaced an infinitely small magnitude from an equilibrium state tend to return to it", if and only if the roots of the secular equation are real, negative and distinct, $d$ 'Alembert is transferring to the roots of the secular equation his conclusions regarding the roots of the equation of the pendulum. In effect, a double root of Eq. (*) of a differential equation of order 2 gives rise to solutions of the form $A t \sin (\alpha t+\beta)$ in which time "comes out of the sine" and implies unbounded oscillations.

In the case of the oscillations of $n$ bodies, the distinction between the two types of equations is more significant: whereas Eq. (*) remains of degree 2, the secular equation passes to degree $n$. Lagrange's criteria for mechanical stability has nevertheless managed the transfer effected by d'Alembert. Now, this time the multiplicity of the roots does not play any role in the stability of the oscillations. The question depends solely on whether it is possible or not to decompose the system (3) into a series of independent equations, an operation today we call a "digitalisation" because it consists in eliminating all the coefficients of the system except those present on its "diagonal". Now, here this reduction is always possible by reason of the symmetry of linear system studied by Lagrange. Each root $\alpha$ of the secular equation generates a number of independent oscillations equal to the order of multiplicity.

This situation might seem contrary to physical intuition. This is one of the mathematical obstacles posed by the generalisation of the notion of proportionality to dimension $n$, that is, to the notion of linearity. Lagrange not only took part in stating this problem, but also in shedding light on certain important properties, such as the role played by the symmetry of linear systems generated by the principles of dynamics.

\subsection{From a common culture to local algebraic practices}

The obstacle of the multiplicity of roots was not overcome until the second part of the nineteenth century, in the works by Sylvester in 1851, by Hermite in 1854, by Karl Weierstrass in 1858 and 1868, and by Camille Jordan in 1870 and 1871 [14], all carried out in direct relation with Lagrange's Mécanique analytique (Fig. 1).

These works gave rise to four distinct lines of research, after a century of discussion about the secular equation. But they also constitute the point of departure for as many different theories: matrix theory, the algebraic theory of quadratic forms, the theory of elementary divisors, and the theory of linear groups. Starting in the 1860s, the common algebraic culture that had been established at the European level around Lagrange's work on the secular equation thus began a progressive process of fragmentation in favour of different local algebraic practices [3].

Nevertheless, up to the end of the nineteenth century, the secular equation continued to serve as the interface 


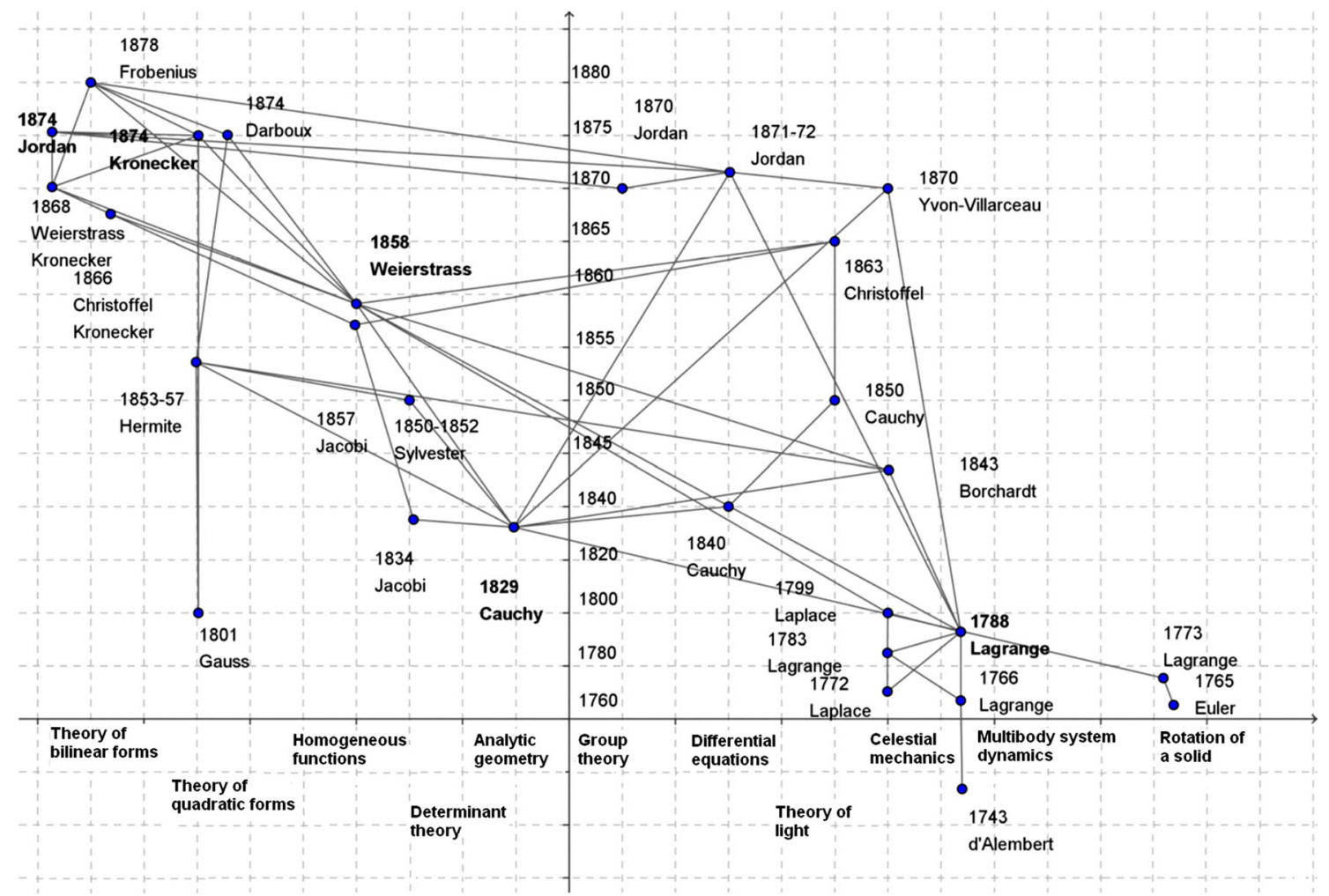

Fig. 1 The network of authors and theories related to Lagrange's Mechanique analytique

between these different practices. In particular, Poincaré referred to it in the 1880s in order to make evident the relationships that existed between the "calculation of Tables", inherited from the work of Hermite, Sylvester's "matrices" and Jordan's "canonical forms". It is in this interface that techniques of linear algebra, such as that of the diagonalisation of matrices, were progressively developed. However, for a long time these procedures were not considered as belonging to an independent theoretical framework. The treatises and handbooks of the nineteenth century only rarely explain them as methods in their own right. This is why the Mécanique analytique remained a primary reference work for such a long time. A direct reading of Lagrange is still at the heart of Poincaré's work on the three-body problem, at the end of the 1880s. A return to Lagrange's approach to linear systems and the secular equation even forms the basis for Poincaré's celebrated work Les méthodes nouvelles de la mécanique céleste:

L'étude des inégalités séculaires par le moyen d'un système d'équations différentielles linéaires à coefficients constants peut donc être regardée comme se rattachant plutôt aux méthodes nouvelles qu'aux méthodes anciennes ([26], p. 2).

(The study of the secular inequalities by means of a system of linear differential equations of constant coefficients can thus be regarded as linked to new methods rather than to old methods).

Like Poincaré, a great number of works at the turn of the nineteenth and twentieth centuries presented the technique of decomposition of linear systems as essential preliminaries to all further development. These procedures are thus progressively placed at the heart of the development of a new discipline, called to play an essential role in the organisation of mathematical knowledge: linear algebra.

\section{Conclusions}

Let us now return to the problems that we mentioned at the beginning of this article regarding the categories used to describe the organisation of knowledge, such as algebra, analysis and mechanics.

In today's terms, Lagrange's works on the secular equation would be inscribed in what we call linear algebra. But this perspective was only imposed in the 1930s. As a consequence it projects conceptual anachronisms on the methods that were being formulated by Lagrange. Thus, the property of "symmetry" of linear systems deriving from mechanics might appear today, stripped of history, almost natural because students learn early on how to envision linear systems from a geometric angle, to speak of 
matrices that are "square", "diagonal", "triangular" or "symmetrical". But we have seen that Lagrange never directly manipulated the "shape" of the linear system in order to reduce it to a diagonal form. His practice was not based on geometric representations. To the contrary, it was a fusion of algebraic and mechanical representations: algebraic factorisation of the secular equation and implicit mechanical representation according to which the small oscillations of a system of $n$ bodies behave as if they were composed of $n$ simple movements.

Further, the use of categories such as linear algebra can also give rise to social anachronisms. Thus, in Lagrange's day, mathematising a set of various mechanical problems through the same analytical expressions was a practice shared by the network of scientists who often held positions in the academies of the great European capitals. Numerous algebraic techniques circulated from one mechanical problem to another thanks to the prizes offered by the academies.

In such a context, it would be useless to isolate the "algebraic" dimension of Lagrange's work from the rest of his scientific output. Furthermore, this algebraic dimension was never limited to the formal character of the manipulation of symbolic expressions. Thus, when the generality so dear to Lagrange was an obstacle to the use of explicit analytical representations - that is, to algebraic formulas-we have seen that Lagrange devised new algebraic techniques based on the decomposition of a linear system and the identification of the property of symmetry.

We have also seen that the works on the secular equation paved the way to specific practices, proper to a particular equation, and very different from the problems of the solvability of general algebraic equations. In the nineteenth century, the "secular equation" itself became a unifying category of a mathematical culture shared at a European level and transversal to different theoretical frameworks. The relationships between astronomy and algebra in the nineteenth century are thus far from being reduced to a back-and-forth between formalisation and application. Not only did specific algebraic techniques emerge from the works on mechanics, but the secular equation also played a role as a shared reference for the entire course of the nineteenth century. This equation provided the basis for the transfer of operating procedures between different domains, a circulation that enriched the procedures with new significations that allowed them to overcome the difficulties posed by multiple roots. The fusion of algebraic and mechanical interpretations that marked Lagrange's approach was finally split by the attribution of geometric, analytic or even arithmetic significations, until Poincaré newly invested it in celestial mechanics at the end of the nineteenth century.
Certain contemporary mathematicians saw in the dislocation of the culture of the secular equation the end of Lagrange's influence. In 1874, a century after Lagrange's works on the secular inequalities, his approach was criticised by Leopold Kronecker as lacking in rigour, because "formal" and falsely general because the too little attention was paid to the particular cases, such as the occurrence of multiple roots. For Kronecker, "true generality" lies in the particular cases, and not in the fact of considering the situations of $n$ variables. During the same period, Lagrange's project of basing the notion of function on the possibility of providing analytical representations was battered by the approach of Georg Cantor, who envisioned a function as an application between two sets.

It was starting in this period that mathematicians began to assign an ambiguous position to Lagrange's works, as representing at once the onset of modernity and the final expression of the eighteenth century. The "algebraic" dimension of these works played an important role in this evaluation. Once the difficulties posed by the occurrence of multiple roots in the secular equation were overcome, and after the theory of sets had been rendered obsolete by the growth in universality of analytical expressions, Lagrange's works were often presented as the culmination of a form of "generic reasoning" that had been developed starting in the sixteen century. This form of reasoning consists in manipulating analytical expressions comprising symbols embodying "general" values, with scant attention paid to the difficulties or inexactitudes that might arise in assigning particular values to those symbols. Lagrange's treatment of small oscillations was considered to be an emblematic example of generic reasoning, because of the slight attention given to the problem posed by the occurrence of multiple roots [12]. More generally, Lagrange's approach was described as "formal" in comparison to the works of the nineteenth century, such as those of Cauchy or Weierstrass [28], who had progressively rendered algebra more rigorous, in particular through their attention to particular cases liable to limit the validity of general algebraic formulas.

This makes is necessary to note that this evaluation of Lagrange's works developed during a period of the fragmentation of the transversal practices brought about by the secular equation, and of the organisation of "algebra" as an independent discipline. This algebra of the late nineteenth century referred to very different significations than those of the algebraic culture constituted by the works of Lagrange; it is hardly surprising that the one criticises the other.

To be sure, such a criticism is never lacking in pertinence when, in retrospect, we situate the works of Lagrange in a general panorama of the long-term evolution of mathematics. But in this article we have placed 
ourselves at a more minute level, that of a problem examined in its technical details. At this level, Lagrange's approach appears far from formal. To the contrary, we have seen the essential role played by the interactions between algebra and mechanics, both in the creation of a new general method and in the discussion of different particular cases. Furthermore, we have shown the fruitfulness of Lagrange's work on the secular equation, which supported analogies between different domains of the mathematical sciences and which were then enriched with new significations throughout the nineteenth century.

(Translated from the French by Kim Williams).

\section{References}

1. Barroso-Filho, W., Comte, C.: La formalisation de la dynamique par Lagrange: L'introduction du Calcul des Variations et l'Unification à partir du Principe de moindre Action. In: Rashed, R., (ed) Sciences à l'Epoque de la Révolution, pp. 329-348. Blanchard, Paris (1988)

2. Brechenmacher, F.: L'identité algébrique d'une pratique portée par la discussion sur l'équation à l'aide de laquelle on détermine les inégalités séculaires des planètes (1766-1874). Sciences et techniques en perspective 1, 5-85 (2007)

3. Brechenmacher, F.: Une histoire de l'universalité des matrices mathématiques. Revue de Synthèse 4, 569-603 (2010)

4. Brechenmacher, F.: The algebraic cast of Poincaré's Méthodes nouvelles de la mécanique céleste. http://hal.archives-ouvertes.fr/ hal-00821686

5. Cauchy, A.-L.: Sur l'équation à l'aide de laquelle on détermine les inégalités séculaires des mouvements des planètes. Exercices de mathématiques. Rpt. in Oeuvres complètes, série 2, tome 9, 4 ,174-195 (1829)

6. Dahan Dalmedico, A.: Le formalisme variationnel dans les travaux de Lagrange. Atti della Accademia della Scienze di Torino 124, 81-206 (1990)

7. Dalmedico, Dahan: Mathématisations. Augustin-Louis Cauchy et l'Ecole Française. Blanchard, Paris (1992)

8. D'Alembert, J.: Traité de dynamique. Chez David, Paris (1743)

9. D'Alembert, J.: Traité de dynamique, 2nd edn. Chez David, Paris (1758)

10. Gilain, C.: D'Alembert et l'intégration des expressions différentielles à une variable. In: Michel, A., Paty, M., (eds.) Analyse et dynamique. Etudes sur l'œuvre de d'Alembert, pp. 207-235. Presses de l'Université Laval, Québec (2002)

11. Gilain, C.: Mathématiques mixtes et mathématiques pures chez d'Alembert: Le cas des systèmes différentiels linéaires. Archives internationales d'histoire des sciences 58, 99-131 (2008)

12. Hawkins, T.: Weierstrass and the theory of matrices. Arch. Hist. Ex. Sci. 17, 119-163 (1977)

13. Hermite, C.: Extrait d'une lettre à M. Borchardt sur l'invariabilité du nombre des carrés positifs et des carrés négatifs dans la transformation des polynômes homogènes du second degré. Journal für die reine und angewandte Mathematik 53, 271-274 (1857)

14. Jordan, C.: Sur les oscillations infiniment petites des systèmes matériels. Comptes rendus de l'Académie des sciences de Paris 74, 1395-1399 (1872)
15. Lagrange, J.-L.: Solution de différents problèmes de calcul intégral. Miscellanea Taurinensia 3 (1766). Rpt. in [20, I, pp. 471-668]

16. Lagrange, J.-L.: Recherches sur les équations séculaires des mouvements des nœuds, et des inclinaisons des orbites des planètes. Hist. de l'acad. des sciences 177 (1778). Rpt. in [20, VI, pp. 635-709]

17. Lagrange, J.-L.: Théorie des variations séculaires des éléments des planètes; Première Partie. Nouv. mém. de l'acad. des sciences de Berlin, (1783). Rpt. in [20, V, pp. 125-207]

18. Lagrange, J.-L. Théorie des variations séculaires des éléments des planètes; Seconde Partie. Nouv. mém. de l'acad. des sciences de Berlin (1784). Rpt. in [20, vol. V, pp. 201-344]

19. Lagrange, J.-L.: Mécanique analitique, Paris, Desaint (1788). 2nd ed. Mécanique analytique, 2 vols. Paris, Courcier (1811-1815). Rpt. in [20, vols. XI, XII]

20. Laplace, P. S.: Mémoire sur les solutions particulières des équations différentielles et sur les inégalités séculaires des planètes. Mem. de l'acad. des sciences de Paris, Partie I (1775). Rpt. in Laplace, Oeuvres, vol. 8, pp. 325-366

21. Laplace, P. S.: Traité de mécanique céleste, vol. 1, Paris, 1799. Rpt. in Laplace, Oeuvres, vol. 1

22. Laskar, J.: La stabilité du système solaire. In: Dahan Dalmedico, A., Chabert, J.-L., Chemla, K., (eds.) Chaos et déterminisme, Seuil, Paris, pp.170-212 (1992)

23. Panza, M.: The Analytical Foundation of Mechanics of Discrete Systems in Lagrange's Théorie des fonctions analytiques, Compared with Lagrange's Earlier Treatments of this Topic. Historia Scientiarum 44, 87-132 (1991)

24. Panza, M.: The Analytical Foundation of Mechanics of Discrete Systems in Lagrange's Théorie des fonctions analytiques, Compared with Lagrange's Earlier Treatments of this Topic. Historia Scientiarum 45, 181-212 (1992)

25. Panza, M.: The Origins of Analytical Mechanics in 18th century. In: Jahnke, H. N., (ed) A History of Analysis. s.l., pp. 137-153. American Mathematical Society, Washington D.C., and London Mathematical Society, London (2003)

26. Poincaré, H.: Les méthodes nouvelles de la mécanique céleste, vol. 1. Gauthier-Villars, Paris (1892)

27. Sylvester, J.J.: Sur une propriété nouvelle de l'équation qui sert à déterminer les inégalités séculaires des planètes. Nouvelles annales de mathématiques, journal des candidats aux écoles polytechnique et normale, Sér. 1(11), 434-440 (1852)

28. Weierstrass, K. Über ein die homogenen Functionen zweiten Grades betreffendes Theorem. M'ber. Akad. der Wiss. Berlin, pp. 207-220 (1858). Rept. in Weierstrass, Werke, 1, pp. 233-246

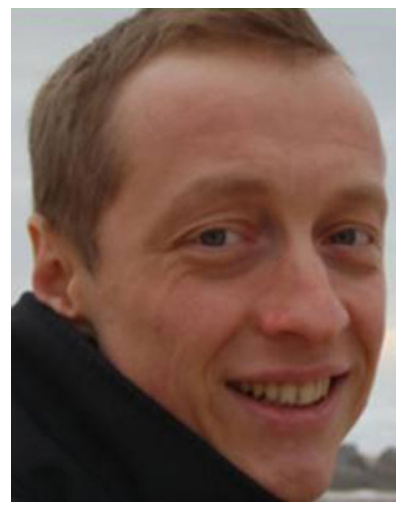

Frédéric Brechenmacher is a lecturer in the history of sciences at the University of Artois (Laboratoire de mathématiques de Lens). He is also a part-time lecturer in the course in history of sciences at the École Polytechnique. His work focuses on the history of algebra from the eighteenth to twentieth centuries. In particular, he is the author of "Une histoire de l'universalité des matrices mathématiques" (Revue de synthèse, 4 (2010), pp. 569-603) and "Self-portraits with Évariste Galois and the shadow of Camille Jordan" (Revue d'histoire des mathématiques, 17 (2011), pp. 271-369). 